\title{
Spontaneous Rupture of the Spleen: An Exceptional Complication of Malaria Due to Plasmodium Ovale. A Case Report
}

\section{DIAI Abdellatif ${ }^{1,3^{*}}$, MAAROUFI Ayoub ${ }^{1,3}$, JEBBAR Nourddine ${ }^{1,3}$, ELKAISSI Jaber ${ }^{1,3}$, Hicham Kechna ${ }^{1,3}$, LAOUTID Jaouad ${ }^{1,3}$} Khalil Mounir ${ }^{2}$

${ }^{1}$ Anesthesiology \&amp; intensive care department, Moulay Ismail Military Hospital, Meknes, Morocco

${ }^{2}$ Anesthesiology \&amp; intensive care department, Military Hospital Mohamed V, RABAT, Morocco

${ }^{3}$ Faculty of Medicine, Sidi Mohamed Ben Abdellah University, Fes, Morocco

DOI: $10.36347 /$ sasjm.2021.v07i02.002

| Received: 26.01.2021 | Accepted: 16.02.2021 | Published: 18.02.2021

*Corresponding author: Diai Abdellatif

Abstract

The diagnosis of Spontaneous rupture of the spleen is sometimes misleading, it is an exceptional complication, it usually occurs in children and the recent expatriate. A 34-year-old Moroccan soldier, assigned to the center of Africa for a humanitarian mission, under chemoprophylaxis based on Mefloquine, was hospitalized in April 2018 for a febrile syndrome with a sensitive abdomen. After initiation of Mefloquine therapy, he developed hemodynamic instability related to intra-abdominal bleeding related to spontaneous rupture of the spleen due to plasmodium oval, which required hemostatic splenectomy. The outcome was favorable after surgery. Spontaneous splenic rupture is the prerogative of Plasmodium vivax and falciparum; about twenty cases have been reported in the literature. Conservative treatment helps preserve the role of the spleen in the immune response, especially in children and people traveling to endemic areas.

Keywords: Spontaneous Rupture Spleen Malaria Plasmodium.

Copyright $\left({ }^{\circ} 2021\right.$ The Author(s): This is an open-access article distributed under the terms of the Creative Commons Attribution 4.0 International License (CC BY-NC 4.0) which permits unrestricted use, distribution, and reproduction in any medium for non-commercial use provided the original author and source are credited.

\section{INTRODUCTION}

Spontaneous rupture of the spleen is a rare and serious complication, probably underestimated in endemic areas [1]. It usually occurs in children and recent expats. The pathophysiological mechanisms are mainly mechanical $[2,3]$. We present the case of a spontaneous splenic rupture during a malaria attack by Plasmodium ovale in a recent expatriate.

\section{PATIENT AND OBSERVATION}

15 days after returning from a six-month stay in central Africa, a 34-year-old Moroccan man with no particular pathological history on chemoprophylaxis based on Mefloquine, consulted in April 2018 for a febrile syndrome at $39 \circ \mathrm{C}$ with chills, sweating profuse, vomiting and abdominal pain. On examination, the patient was conscious, hemodynamically and respiratory stable. The examination finds a tender flexible abdomen without splenomegaly or hepatomegaly.
An abdominal ultrasound was performed, revealing an abdominal effusion of moderate abundance. The complete blood count showed hemoglobin at $15.4 \mathrm{~g} / \mathrm{dl}$, platelets at $539 \mathrm{G} / 1$ and white blood cells at 19G / 1. The blood smear and the thick film revealed trophozoites, gametocytes, and oval plasmodium schizonts with a parasitaemia rate of $0.01 \%$.

A treatment with "Lumate forte" 1cp / 12h was started. The evolution was marked by a clinical worsening with the onset of a state of shock, an increasingly painful abdomen, a decrease in the hemoglobin level to $9.6 \mathrm{~g} / \mathrm{dl}$, platelets to $94,000 \mathrm{~g} / \mathrm{l}$ and leukocytes at $7510 \mathrm{~g} / \mathrm{l}$, led us to redo an ultrasound which revealed an abundant abdominal effusion. An exploratory laparotomy performed revealed an abundant hemoperitoneum secondary to a rupture of the spleen requiring a hemostatic splenectomy. 

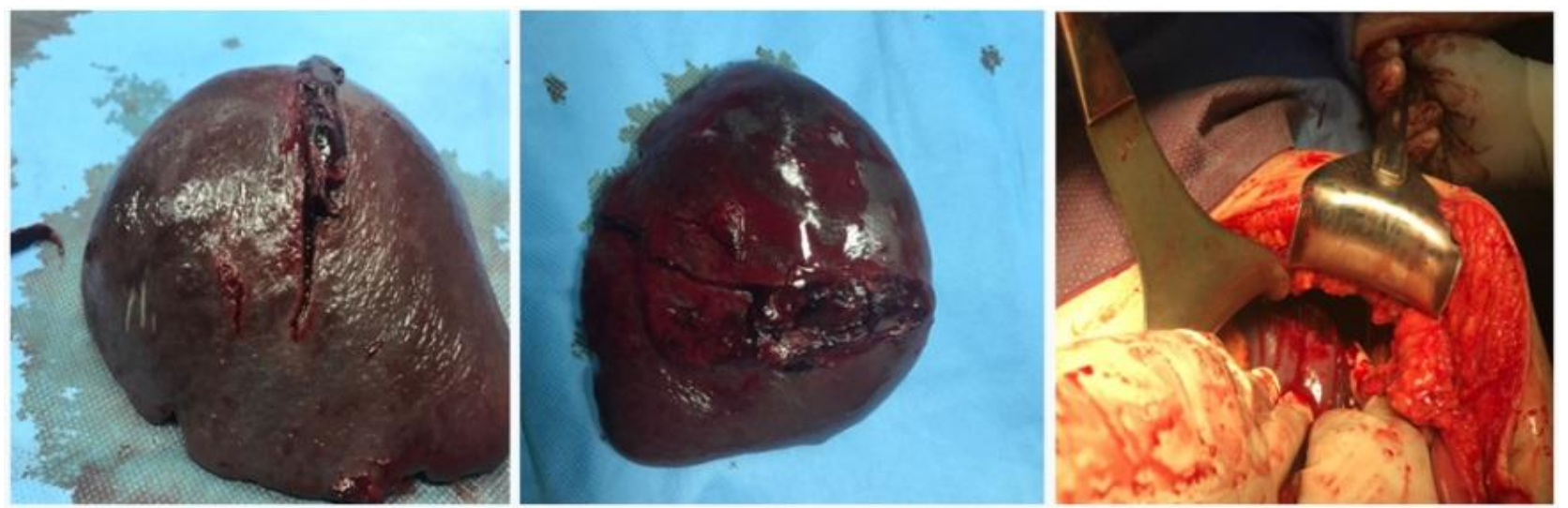

Fig-1: Images of spontaneous rupture of the spleen

The outcome was favorable after a two-day stay in intensive care with regression of fever and abdominal signs and hemodynamic stabilization, after monitoring and strict bed rest. Pathological examination found brownish malarial pigments confirming the etiology of malaria.

\section{DISCUSSION}

In malaria, splenomegaly results from the hemodestructive role of the spleen by the accumulation of elements of phagocytosis and debris from the bursting of rosaceous bodies. It is common in children and expatriates in an endemic area with a prevalence of 50 to $80 \%[1,4]$.

Spontaneous rupture is due to the fragility of the spleen during an acute attack or primary invasion or during progressive visceral malaria $[5,6]$. The prevalence of ruptured spleen in malaria is $0-2 \%$ [3]. The difficulty of accessing healthcare in several malaria-endemic countries makes its frequency underestimated, which explains the few cases reported by African doctors.

P. vivax and falciparum are the species reported in the majority of cases. $\mathrm{P}$. vivax is responsible for a weak but prolonged parasitism involved in weakening of the spleen $[5,6]$. But all Plasmodium species can be incriminated [5].

\section{Three mechanisms seem to play a role in this process}

- Cellular hyperplasia and engorgement lead to an increase in intrasplenic tension.

- Compression by the abdominal muscles during coughing, sneezing, or defecation;

- Vascular occlusion due to hyperplasia of the endothelial reticulum responsible for infarction, whether or not associated with subcapsular hematoma [7].

Twisting of the pedicle is possible in splenomegaly in progressive visceral malaria [6]. Positive pressure mechanical ventilation seems to play a role in some observations [3]. The rupture of the spleen can occur in one step as is the case with our patient or in two steps after the appearance of a subcapsular hematoma. Clinically, shock with acute anemia is the dramatic picture of rupture, and the occurrence of even moderate abdominal pain during a bout of malaria deserves attention.

Abdominal ultrasound is usually a quick diagnosis. The abdominal scan is the gold standard. Splenectomy is the radical treatment for spontaneous ruptures of the spleen. However, conservative treatment can be offered which consists of clinical and haematological monitoring during a 15-day hospitalization with strict bed rest and blood transfusion as needed, given the morbidity of splenectomy, and the role of spleen in the immune response [2-4, 7-9]. This therapeutic attitude is justified in children and people who regularly travel to endemic areas $[4,9]$.

Somatostatin was proposed by some authors to reduce splanchnic flow in ruptures of normal spleens [10]. Splenectomy should be reserved for severe ruptures of the spleen or those with prolonged or recurrent bleeding [5]. Reconstructive surgery of the spleen with partial resections is preferred [8]. Parasitological examinations following splenectomy are justified when drug-resistant malaria is suspected [6].

\section{CONCLUSION}

Spontaneous rupture of the spleen is a diagnostic and therapeutic emergency, despite its rarity, it must be considered when faced with any abdominal pain associated or not with haemodynamic instability in patients treated for malaria. Its therapeutic management involves the selection of patients who can benefit from conservative treatment and splenectomy as a last resort.

\section{REFERENCES}

1. Patel MI. Spontaneous rupture of a malarial spleen. Med J Aust. 1993; 159:836-7.

2. Ciezy JKA Richens JE. Nonoperative management of spontaneously ruptured malarial spleen. $\mathrm{Br} \mathrm{J}$ Surg. 1985; 72:990. 
3. Jacobs FM, Prat D, Petit F, Smadja C, Brivet FG. Spontaneous rupture of the spleen during malaria: a conservative treatment approach may be appropriate. Clin Infect Dis. 2005; 40:1858-9.

4. Yagmur Y, Hamidi Kara I, Aldemir M, Büyükbayram H, Tacyildiz IH, Keles C. Spontaneous rupture of malarial spleen: two case reports and review of literature. Crit care. 2000; 5:309-13.

5. Zingman BS, Viner BL. Splenic complications in malaria: case report and review. Clin Infect Dis. 1993; 16:223-32.

6. Touze JE, Martet G, Garnotel E, Beauté D, Ducourau JP, Raphenon G. Une complication inhabituelle du paludisme à Plasmodium falciparum chloroquino-résistant: la rupture splénique spontanée. Ann Med Interne. 1989; 140:228-9.

7. Mokashi AJ, Shirahatti RG, Prabhu SK, Vagholkar KR. Pathological rupture of malarial spleen. J Postgrad Med 1992; 38:141-2.

8. Imbert P, Rapp C, Debord T. Spontaneous rupture of the spleen during malaria: a conservative treatment is appropriate for selected patients. Clin Infect Dis. 2006; 42:1207-8.

9. Hamel CT, Blum J, and Harder F, Kocher T. Nonoperative treatment of splenic rupture in malaria tropica: review of literature and case report. Acta Trop. 2002; 82:1-5.

10. Orloff MJ, PeskinGW. Spontaneous rupture of the normal spleen a surgical enigma. Surg Gynecol Obstet. 1985; 106:1-11. 